\section{Practical Formulation of the Relation Between Filter Specifications and the Requirements for Integrator Circuits}

\author{
W. J. A. DE HEIJ, E. SEEVINCK, AND K. HOEN
}

Abstract - The design of integrated high-frequency continuous-time filters has made considerable progress in the past few years. As the signal frequencies increase the design of the integrator circuits used in most of these filters becomes more critical. To give direction to the circuit design minimum specifications for the gain and phase of the integrator circuits would be helpful. In this paper a practical method of finding these integrator specifications from the filter specifications is developed. An example of application of the method to a sixth-order Chebyshev band-pass filter is given and the result is verified by computer simulation.

\section{INTRODUCTION}

During the past few years there have been a number of publications on fully integrated continuous-time filters operating at high frequencies [1]-[8]. Except for [8] the basic building block of these filters is the integrator. The gyrator resonance circuits used in [5] and [6] can be considered as a pair of cross-coupled integrators.

An important problem in high-frequency filtering is the accurate realization of the specified poles and/or zeros of the filter. The quality factor $Q$ of poles and zeros is highly sensitive to the phase of the integrators at the pole and zero frequencies. Therefore, the circuit designer should have accurate specifications for the gain and phase of integrator circuits.

In this paper a method to find the integrator specifications from filter specifications is developed from an analysis of the influence of nonideal integrators on the positions of poles and zeros of a filter. In Section II a simple model for the behavior of practical integrators is formulated. In Section III it is shown that the influence of nonideal integrators on the filter transfer function is independent of the filter topology. In Section IV the integrator model is used to study the influence of the non-ideal integrator on biquad transfer functions. In Section V the theory is applied to an example. Conclusions are given in Section VI.

\section{THE INTEgRator MODEL}

For the analysis of the effect of nonideal integrators on filter transfer functions we need a model of the nonideal integrator. In Fig. 1 the magnitude and phase of an ideal integrator $H_{\mathrm{id}}(s)$ and a practical (nonideal) integrator $H_{\mathrm{ni}}(s)$ are shown.

The ideal integrator in Fig. 1 has the following transfer function:

$$
H_{\mathrm{id}}(s)=\frac{\omega_{0}}{s}
$$

For angular frequencies up to $\approx 0.2 * \omega_{p 2}$ the transfer function

Manuscript received July 29, 1988; revised December 22, 1988. This paper was recommended by Associate Editor T. T. Vu.

W. J. A. de Heij and K. Hoen are with the Department of IC-Technology and Electronics, Faculty of Electrical Engineering, University of Twente, 7500 AE Enschede, The Netherlands.

E. Seevinck is with the Department of IC-Technology and Electronics, Faculty of Electrical Engineering, University of Twente, 7500 AE Enschede, The Netherlands, and also with Philips Research Laboratories, 5600 JA Eindhoven, The Netherlands.

IEEE Log Number 8928278 .
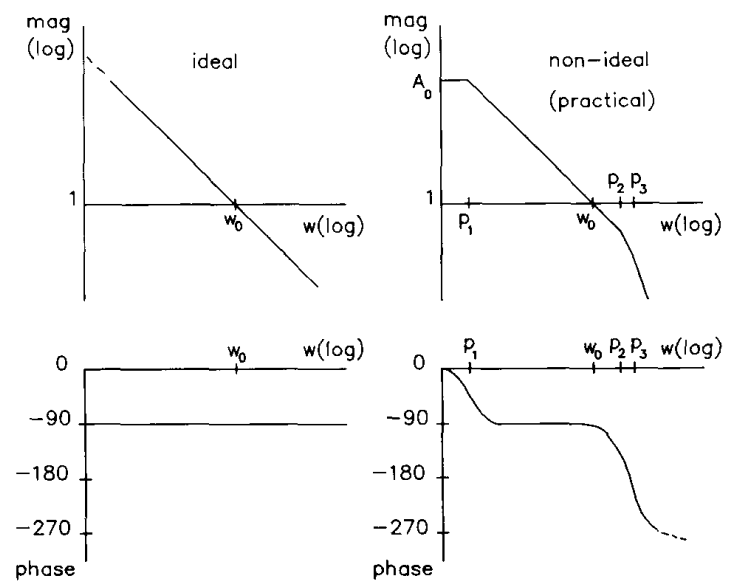

Fig. 1. Gain and phase of an ideal integrator and a non-ideal integrator.

$H_{\mathrm{ni}}(s)$ of the practical integrator can be approximated by

$$
H_{\mathrm{ni}}(s)=\frac{A_{0}}{\left(1+s \tau_{1}\right)\left(1+s \tau_{2}\right)} .
$$

Time constant $\tau_{1}=\tau_{p 1}$ belongs to the dominant pole $p_{1}$. The dc-gain is equal to $A_{0}$. The unity gain frequency $\omega_{0}$ is well approximated by $\omega_{0} \approx A_{0} / \tau_{1}$ if $1 / \tau_{1} \ll \omega_{0} \ll 1 / \tau_{2}$. Time constant $\tau_{2}$ is used to approximate the phase shift due to the parasitic poles and zeros which are usually at frequencies much higher than the signal frequencies processed by the filter. Therefore, in our analysis $\left|s \tau_{2}\right| \ll 1$ will hold and we can write (2) as

$$
H_{n i}(s)=A_{0} \frac{1-s \tau_{2}}{1+s \tau_{1}}
$$

Model (3) is used in Section IV because it is mathematically easier to handle than model (2) [10]. To find an appropriate value for $\tau_{2}$ we use the quality factor of an integrator which is easily defined if we write its transfer function $(s=j \omega)$ as follows:

$$
H_{\mathrm{int}}(j \omega)=\frac{1}{R(\omega)+j X(\omega)} .
$$

Then the quality factor of the integrator can be defined as [11]:

$$
Q_{\text {int }}(\omega)=\frac{X(\omega)}{R(\omega)}=\tan \left(-\arg \left(H_{\mathrm{int}}(j \omega)\right)\right)
$$

For the integrator models (2) and (3) the reciprocal of the quality factor $\left(\tau_{1} \gg \tau_{2}\right)$ can be written as

$$
\frac{1}{Q_{\text {int }}(\omega)}=\frac{1-\omega^{2} \tau_{1} \tau_{2}}{\omega\left(\tau_{1}+\tau_{2}\right)} \approx \frac{1}{\omega \tau_{1}}-\omega \tau_{2}
$$

To model a practical integrator we first calculate $\tau_{1}$ from the dominant pole frequency. Next the quality factor $Q_{\text {int }}\left(\omega_{0}\right)$ is found from the phase at the unity-gain frequency $\omega_{0}$ using (5). Finally, $\tau_{2}$ is calculated from $Q_{\text {int }}\left(\omega_{0}\right)$ and $\tau_{1}$ using (6). This approximation by model ( 3 ) will be useful up to $\approx 0.2 * \omega_{p 2}$ (Fig. 1). Usually this frequency range covers the signal frequencies where the performance of the filter employing the integrators is critical. 


\section{The INDEPENDENCE OF THE EFFECT OF NONIDEAL INTEGRATORS OF THE FILTER TOPOLOGY}

In this section it will be shown that the effect of nonideal integrators on the transfer function of a filter is independent of the filter topology in which the integrators are used.

Let the following normalized filter transfer function be specified:

$$
H(S)=\frac{a_{m} S^{m}+a_{m-1} S^{m-1}+\cdots+a_{1} S+a_{0}}{b_{n} S^{n}+b_{n-1} S^{n-1}+\cdots+b_{1} S+b_{0}}, \quad S=\frac{s}{\omega_{0}} .
$$

The specified $H(S)$ can be realized by many filter topologies. In these topologies ideal integrators $H_{\text {id }}(S)=1 / S$ are interconnected in different ways via multiplication of signals by constants $K_{1}, K_{2}, \cdots, K_{N}$ and summation of signals, to form a network [9] The constants are functions of the filter coefficients $a_{i}$ and $b_{j}$ :

$$
K_{h}=K_{h}\left(a_{0}, a_{1}, \cdots, a_{m}, b_{0}, b_{1}, \cdots, b_{n}\right), \quad h=1,2, \cdots, N .
$$

The form of the functions and the number of constants $N$ is topology dependent, causing for example the difference in sensitivity between filter topologies.

If the numerator and the denominator of (7) are divided by the highest power of $S$ and the ideal integrator given by (1) is inserted with unity-gain frequency $\omega_{0}$ we obtain the following expression for the case $m \leqslant n$ (the case $m>n$ is not essentially different):

TABLE I

CoffFicients of Biouad Transfer Functions

\begin{tabular}{lccccc}
\hline \hline Function & $a_{2}$ & $a_{1}$ & $a_{0}$ & $b_{1}$ & $b_{0}$ \\
\hline Low pass & 0 & 0 & $K \Omega_{p}^{2}$ & $\Omega_{p} / Q_{p}$ & $\Omega_{p}^{2}$ \\
Band pass & 0 & $H_{B} \Omega_{p} / Q_{p}$ & 0 & $\Omega_{p} / Q_{p}$ & $\Omega_{p}^{2}$ \\
High pass & $K$ & 0 & 0 & $\Omega_{p} / Q_{p}$ & $\Omega_{p}^{2}$ \\
Notch & $K$ & 0 & $K \Omega_{z}^{2}$ & $\Omega_{p} / Q_{p}$ & $\Omega_{p}^{2}$ \\
All pass & $K$ & $-K \Omega_{p} / Q_{p}$ & $K \Omega_{p}^{2}$ & $\Omega_{p} / Q_{p}$ & $\Omega_{p}^{2}$ \\
\hline
\end{tabular}

most simple approach because complex pole and zero pairs can not be described with transfer functions with a degree lower than two. High order filters can be considered as a cascade of firstand second-order blocks enabling the analysis of the effect of nonideal integrators on the filter transfer function to be broken up into smaller problems. As a consequence of the argument in Section III the results will be valid independently of the actual topology used to realize the filter transfer function $H(S)$.

A general normalized biquad transfer function with complex poles and zeros is given by

$$
H_{b}(S)=\frac{N(S)}{D(S)}=\frac{a_{2} S^{2}+a_{1} S+a_{0}}{S^{2}+b_{1} S+b_{0}}, \quad S=\frac{s}{\omega_{0}} .
$$

$$
H(s)=\frac{a_{m} H_{\mathrm{id}}^{n-m}(s)+a_{m-1} H_{\mathrm{id}}^{n-m+1}(s)+\cdots+a_{1} H_{\mathrm{id}}^{n-1}(s)+a_{0} H_{\mathrm{id}}^{n}(s)}{b_{n}+b_{n-1} H_{\mathrm{id}}(s)+\cdots+b_{1} H_{\mathrm{id}}^{n-1}(s)+b_{0} H_{\mathrm{id}}^{n}(s)} .
$$

Note that in (9) identical integrators are assumed

If different filter topologies realize $H(s)$ (9) with ideal integrators $H_{\text {id }}(s)$ they must produce the same set of filter coefficients $a_{i}$ and $b_{j}$ each through the inverse of its own set of functions (8). We can take any one of these filter topologies or networks and replace $H_{\mathrm{id}}(s)$ with a nonideal integrator $H_{\mathrm{ni}}(s)$ modeled by (3) having unity-gain frequency $\omega_{0}$ without changing the network structure nor the constants $K_{1}, K_{2}, \cdots, K_{N}$. Then the replacement results in a modified transfer function given by
There are five types of biquad transfer functions: low pass, band pass, high pass, notch or band reject, and all pass. The coefficients $a_{i}$ and $b_{j}$ of (11) for these transfer functions are given in Table I.

In Table I $\Omega_{z}=\omega_{z} / \omega_{0}$ and $\Omega_{p}=\omega_{p} / \omega_{0}$ are normalized frequencies. $K$ and $H_{B}$ are gain constants.

In the following analysis the ideal integrators $H_{\mathrm{id}}(S)$ are replaced by nonideal integrators $H_{\mathrm{ni}}(S)$ as described by (3)

$$
H^{\prime}(s)=\frac{a_{m} H_{\mathrm{ni}}^{n-m}(s)+a_{m-1} H_{\mathrm{ni}}^{n-m+1}(s)+\cdots+a_{1} H_{\mathrm{ni}}^{n-1}(s)+a_{0} H_{\mathrm{ni}}^{n}(s)}{b_{n}+b_{n-1} H_{\mathrm{ni}}(s)+\cdots+b_{1} H_{\mathrm{ni}}^{n-1}(s)+b_{0} H_{\mathrm{ni}}^{n}(s)} .
$$

Because the network structure and the constants $K_{1}, K_{2}, \cdots, K_{N}$ remain unchanged and $H_{\text {id }}(s)$ and $H_{\text {ni }}(s)$ have the same unity gain frequency the difference between $H(s)$ and $H^{\prime}(s)$ is only due to the nonideal behavior of $H_{\mathrm{ni}}(s)$ compared to $H_{\mathrm{id}}(s)$. We have to design our integrator circuits (which will always be non-ideal) in such a way that the difference between $H^{\prime}(s)$ and the specified transfer function $H(s)$ is kept within acceptable limits.

By the argument above it is intuitively shown that $H^{\prime}(s)$ is found from the specified filter transfer function $H(S)$ independently of the filter topology. So we can conclude that the effect of nonideal integrators can be analyzed independently of the filter topology. This result will be used in the next section to simplify the analysis.

\section{INFLUENCE OF NONIDEAL INTEGRATORS ON BIQUAD Transfer Functions}

In this section the influence of nonideal integrators on firstand second-order transfer functions will be analyzed. It is the having the same unity-gain frequency $\Omega_{0}=1$ :

$$
H_{\mathrm{ni}}(S)=A_{0} \frac{1-S T_{2}}{1+S T_{1}} .
$$

In (12) the time constants $\tau_{1}$ and $\tau_{2}$ of (3) are normalized with respect to $\omega_{0}$ :

$$
T_{1}=\tau_{1} \omega_{0}=A_{0} \quad \text { and } \quad T_{2}=\tau_{2} \omega_{0}=\frac{A_{0}}{\tau_{1}} \tau_{2} .
$$

The replacement results in a modified transfer function $H_{b}^{\prime}(S)$ :

$$
H_{b}^{\prime}(S)=\frac{N^{\prime}(S)}{D^{\prime}(S)}=\frac{a_{2}^{\prime} S^{2}+a_{1}^{\prime} S+a_{0}^{\prime}}{S^{2}+b_{1}^{\prime} S+b_{0}^{\prime}} .
$$

The modified coefficients $a_{i}^{\prime}$ and $b_{j}^{\prime}$ are given in Appendix A. They are derived under the assumption that $\Omega_{0}$ is well approximated by $\Omega_{0}=A_{0} / T_{1}$ (Section II)

The influence of nonideal integrators on any type of biquad transfer function can be analyzed by substitution of the coeffi- 
cients from Table I into (A1)-(A5) in Appendix A. The case of the first-order transfer function is treated in Appendix $\mathrm{A}$.

All biquad transfer functions have the same denominator polynomial $D(S)$. For the coefficients $b_{j}^{\prime}$ we find

$$
\begin{aligned}
& b_{1}^{\prime}=\frac{\frac{2}{T_{1}}+\left(1-\frac{T_{2}}{T_{1}}\right) \frac{\Omega_{p}}{Q_{p}}-2 T_{2} \Omega_{p}^{2}}{1-\frac{\Omega_{p}}{Q_{p}} T_{2}+\Omega_{p}^{2} T_{2}^{2}} \\
& b_{0}^{\prime}=\frac{\frac{1}{T_{1}^{2}}+\frac{\Omega_{p}}{Q_{p} T_{1}}+\Omega_{p}^{2}}{1-\frac{\Omega_{p}}{Q_{p}} T_{2}+\Omega_{p}^{2} T_{2}^{2}} .
\end{aligned}
$$

Using Table I we can calculate the biquad parameters $\Omega_{p}^{\prime}$ and $Q_{p}^{\prime}$ for nonideal integrators from $b_{0}^{\prime}$ and $b_{1}^{\prime}$ and simplify the result using the fact that for most known integrator designs $T_{1} \gg 1$ and $T_{2} \ll 1$. Using (6) and (13) the result can be expressed in the quality factor $Q_{\text {int }}(\Omega)$ of the integrator:

$$
\begin{aligned}
& \Omega_{p}^{\prime}=\sqrt{b_{0}^{\prime}} \approx \Omega_{p} \\
& Q_{p}^{\prime}=\frac{\sqrt{b_{0}^{\prime}}}{b_{1}^{\prime}} \approx \frac{\Omega_{p}}{\frac{2}{T_{1}}+\frac{\Omega_{p}}{Q_{p}}-2 T_{2} \Omega_{p}^{2}}=Q_{p} \frac{1}{1+\frac{2 Q_{p}}{Q_{\text {int }}\left(\Omega_{p}\right)}} .
\end{aligned}
$$

At a frequency $\Omega=(1+\alpha) \Omega_{p}$ the ratio of $\left|D^{\prime}(j \Omega)\right|$ and $|D(j \Omega)|$ becomes equal to

$$
\frac{\left|D^{\prime}\left(j(1+\alpha) \Omega_{p}\right)\right|}{\left|D\left(j(1+\alpha) \Omega_{p}\right)\right|}=\frac{\sqrt{\alpha^{2}(\alpha+2)^{2}+\frac{(1+\alpha)^{2}}{Q_{p}^{\prime 2}}}}{\sqrt{\alpha^{2}(\alpha+2)^{2}+\frac{(1+\alpha)^{2}}{Q_{p}^{2}}}}
$$

Using (18) we find from (19) for $\Omega=\Omega_{p}$ :

$$
\frac{\left|D^{\prime}\left(j \Omega_{p}\right)\right|}{\left|D\left(j \Omega_{p}\right)\right|}=1+\frac{2 Q_{p}}{Q_{\text {int }}\left(\Omega_{p}\right)} .
$$

Equations (18) and (20) are important from a practical point of view because $Q_{\text {int }}\left(\Omega_{p}\right)$ is simply related to the phase of the integrator at $\Omega_{p}$ by (5). The biquad transfer functions in Table I have different numerators $N(S)$. They will be treated briefly below.

- Low pass: $N(S)=K \Omega_{p}^{2}$

$$
K^{\prime}=a_{0}^{\prime} / b_{0}^{\prime} \approx K \text {. }
$$

- Band pass: $N(S)=H_{B}\left(\Omega_{p} / Q_{p}\right) S$

$$
H_{B}^{\prime}=a_{1}^{\prime} / b_{1}^{\prime} \approx H_{B} \frac{1}{1+\frac{2 Q_{p}}{Q_{\mathrm{int}}\left(\Omega_{p}\right)}} .
$$

Magnitude at dc:

$$
\left|H_{h}(j 0)\right|=a_{0}^{\prime} / b_{0}^{\prime}>0 .
$$

- All pass: $N(S)=K\left(S^{2}+\left(\Omega_{z} / Q_{z}\right) S+\Omega_{z}^{2}\right)$ with $Q_{z}=-Q_{p}$ and $\Omega_{z}=\Omega_{p}$

$$
\begin{aligned}
& K^{\prime}=a_{2}^{\prime} \approx K \\
& Q_{z}^{\prime}=\frac{\sqrt{a_{0}^{\prime} a_{2}^{\prime}}}{a_{1}^{\prime}} \approx Q_{z} \frac{1}{1+\frac{2 Q_{z}}{Q_{\text {int }}\left(\Omega_{z}\right)}} \\
& \Omega_{z}^{\prime}=\sqrt{a_{0}^{\prime} / a_{2}^{\prime}} \approx \Omega_{z} .
\end{aligned}
$$

- High pass: $N(S)=K S^{2}$

$K^{\prime}$ is given by (24).

$$
\left|H_{b}(j 0)\right|=a_{0}^{\prime} / b_{0}^{\prime}>0 .
$$

- Notch: $N(S)=K\left(S^{2}+\Omega_{z}^{2}\right)$

$K^{\prime}$ and $\Omega_{z}^{\prime}$ are given by (24) and (26).

According to (25) $Q_{z}^{\prime}$ becomes equal to

Magnitude at $\Omega_{z}$ :

$$
Q_{z}^{\prime} \approx 0.5 * Q_{\text {int }}\left(\Omega_{z}\right) \text {. }
$$

$$
\left|H_{b}^{\prime}\left(j \Omega_{z}\right)\right| \approx \frac{\left|\frac{2 K \Omega_{z}^{2}}{Q_{\mathrm{int}}\left(\Omega_{z}\right)}\right|}{\sqrt{\left(\Omega_{p}^{2}-\Omega_{z}^{2}\right)^{2}+\Omega_{p}^{2} \Omega_{z}^{2}\left(1 / Q_{p}+2 / Q_{\mathrm{int}}\left(\Omega_{p}\right)\right)^{2}}} .
$$

From (21) to (29) we can find for low-pass, band-pass, and high-pass biquads:

$$
\frac{\left|H_{b}(j \Omega)\right|}{\left|H_{b}^{\prime}(j \Omega)\right|} \approx \frac{\left|D^{\prime}(j \Omega)\right|}{|D(j \Omega)|} .
$$

The right-hand side of (30) is given by (19) and (20). For the notch and all-pass biquads also changes of the numerator have to be taken into account, using (19) with $\Omega=(1+\beta) \Omega_{z}, Q_{z}$ and $Q_{z}^{\prime}$. We can conclude that with use of model (3) for nonideal integrators simple and practically useful expressions for the effect of nonideal integrators on the magnitude of second-order transfer functions can be derived. The effect on phase can also be studied using the general equations of Appendix A. In the expressions the quality factor of the integrator which is related to the phase of the integrator by (5) is the key parameter, making the expressions particularly useful for practical calculations. The theory in this section can be used to find integrator specifications from a desired filter response. In the next section an example will be given.

\section{Application to a Sixth-Order Chebyshev BAND-PASS FILTER}

In this section it is demonstrated how the results of the previous section can be used to find integrator specifications from the filter specifications. Assume we have the following normalized transfer function of a sixth-order Chebyshev bandpass filter with $0.5-\mathrm{dB}$ ripple:

$$
H(S)=\frac{K S^{3}}{\left(S^{2}+0.05627 S+0.8154\right)\left(S^{2}+0.1253 S+1\right)\left(S^{2}+0.06902 S+1.226\right)} .
$$


TABLE II

Biquad Parameters $(j=1,2,3)$

\begin{tabular}{ccrc}
\hline \hline & $H_{B_{j}}$ & \multicolumn{1}{c}{$Q_{p j}$} & $\Omega_{p j}$ \\
\hline$H_{b 1}(S)$ & 3.1793 & 16.04 & 0.9030 \\
$H_{b 2}(S)$ & 1.4279 & 7.98 & 1.0000 \\
$H_{b 3}(S)$ & 2.5920 & 16.04 & 1.1072 \\
\hline
\end{tabular}

TABLE III

\begin{tabular}{ccc}
\multicolumn{2}{c}{ RESULTS FOR $Q_{\text {int }}\left(\Omega_{p j}\right)$ FROM $(32)$} \\
\hline \hline & $0 \cdots+0.5 \mathrm{~dB}$ dev. & $-0.5 \cdots 0 \mathrm{~dB}$ dev. \\
& $Q_{\text {int }}\left(\Omega_{p j}\right)$ & $Q_{\text {int }}\left(\Omega_{p j}\right)$ \\
\hline$H_{b 1}(\Omega)$ & $-\infty \cdots-573.5$ & $541.4 \cdots \infty$ \\
$H_{b 2}(\Omega)$ & $-\infty \cdots-285.3$ & $269.4 \cdots \infty$ \\
$H_{b 3}(\Omega)$ & $-\infty \cdots-573.5$ & $541.4 \cdots \infty$ \\
\hline
\end{tabular}

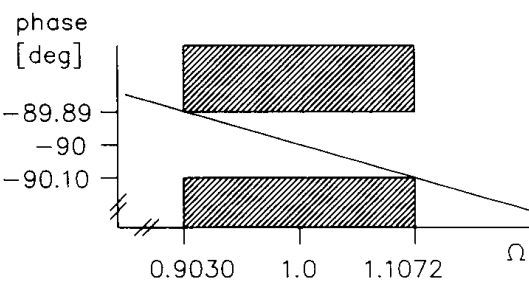

Fig. 2. Integrator phase specification for the sixth-order Chebyshev bandpass filter.

We want to find integrator specifications if the deviation from the ideal response may not exceed $0.5 \mathrm{~dB}$ over the passband. We can treat $H(S)$ as a cascade of three band-pass biquads $H_{b 1}(S) * H_{b 2}(S) * H_{b 3}(S)$. The parameters of the biquads are given in Table II. We will use (17)-(20). Because band-pass biquads satisfy (30) we can use (20) to find that the response deviation of $\pm 0.5 \mathrm{~dB}$ at the pole frequency of a single biquad results in

$$
Q_{\text {int }}\left(\Omega_{p j}\right)=\frac{2 Q_{p j}}{10^{\mp 0.025}-1} .
$$

This equation gives two minimum $Q$-factors (considering absolute values) for each biquad. The interpretation of the result is given in Table III. Using (5) we find that the phase of the integrator over the passband must be between $-89.89^{\circ}$ and $-90.10^{\circ}$. A useful phase specification is shown in Fig. 2.

The quality factor of the integrator is given by (6) and (13):

$$
\frac{1}{Q_{\text {int }}(\Omega)}=\frac{1}{\Omega T_{1}}-\Omega T_{2}
$$

We can solve for $T_{1}$ and $T_{2}$ at frequencies $\Omega_{p 1}=0.9030$ and $\Omega_{p 3}=1.1072$ considering the maximum allowable phase error as shown in Fig. 2. We then find

$$
T_{1}=113.4 \text { and } T_{2}=8.769 * 10^{-3} \text {. }
$$

These are minimum specifications. Larger $T_{1}$ and smaller $T_{2}$ result in better integrators. Let the actual unity gain frequency $\omega_{0}$ in our filter realization be $2 \pi * 500 * 10^{3} \mathrm{rad} / \mathrm{s}$. Then we find the

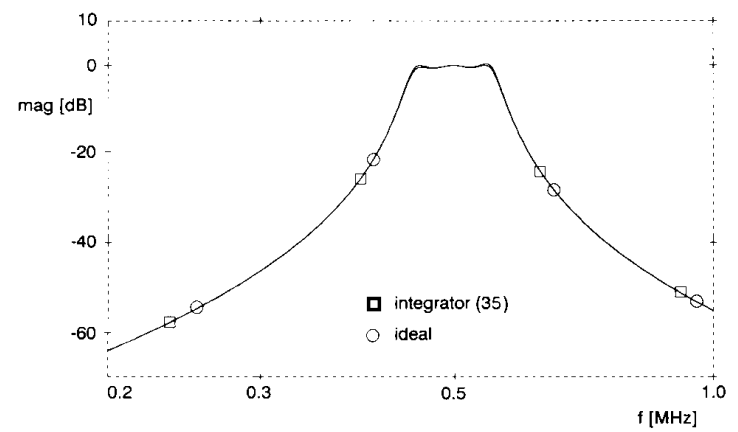

Fig. 3. Magnitude response of bandpass filter with ideal integrators and with integrators specified by (35).

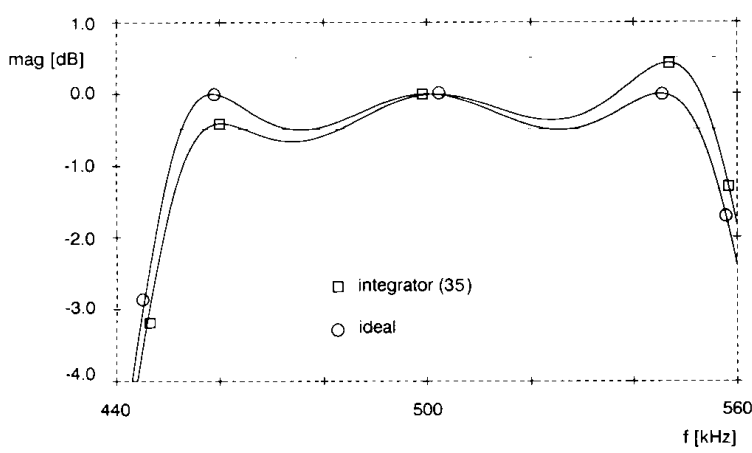

Fig. 4. Detail of passband response from Fig. 3.

following integrator specifications from (34) using (13): dc-gain: 113.4

Dominant pole:

$$
f_{p 1}=\frac{1}{2 \pi \tau_{1}}=4409 \mathrm{~Hz} .
$$

High frequency pole:

$$
f_{p 2}=\frac{1}{2 \pi \tau_{2}}=57.02 \mathrm{MHz} .
$$

In practice one must design the integrator circuits to match the gain and phase following from (35) using passive or active compensation or a $Q$-control loop [1], [4], [7] if necessary.

If we insert the integrator with these specifications in for example a leapfrog topology we obtain a filter response shown in Figs. 3 and 4. Similar simulations for other topologies result in exactly the same transfer functions as was expected from the argument in Section III.

Fig. 3 shows that the stopband response of the filter with integrators as specified by (35) does not significantly differ from the ideal response. Fig. 4 shows that the difference in the passband is within the required $0.5 \mathrm{~dB}$. These results were obtained by PSPICE simulation with integrator models consisting of dependent sources, resistors and capacitors, allowing full control of the parasitic effects being studied.

In the calculation above it is assumed that the deviation of one of the biquads is not noticeable at the pole frequencies of the others. For bandpass biquads this can be checked with (19) and (30). Values of $Q_{p}$ are found in Table II. Values of $Q_{p}^{\prime}$ can be calculated with (18) from (33) and (34). Substituting these values in (19) together with appropriate values of $\alpha$ one can verify that 
the assumption made above indeed results in a very small error in this case.

\section{Conclusions}

The influence of non-ideal integrators on a specified filter transfer function can be analyzed independently of the filter topology using only simple equations in which the quality factor of the integrator is the key parameter. Because the quality factor follows directly from the phase of the integrator as a function of frequency we obtain a practically useful formulation. The example of application demonstrates the possibility to find integrator specifications from the filter specifications. Especially for high frequency continuous-time filters where integrator design is critical it is important to have accurate specifications for the integrator circuits to match.

\section{APPENDIX A}

The modified biquad coefficients in (14):

$$
\begin{aligned}
& a_{2}^{\prime}=\frac{a_{2}-a_{1} T_{2}+a_{0} T_{2}^{2}}{1-b_{1} T_{2}+b_{0} T_{2}^{2}} \\
& a_{1}^{\prime}=\frac{\frac{2 a_{2}}{T_{1}}+\left(1-\frac{T_{2}}{T_{1}}\right) a_{1}-2 T_{2} a_{0}}{1-b_{1} T_{2}+b_{0} T_{2}^{2}} \\
& a_{0}^{\prime}=\frac{\frac{a_{2}}{T_{1}^{2}}+\frac{a_{1}}{T_{1}}+a_{0}}{1-b_{1} T_{2}+b_{0} T_{2}^{2}} \\
& b_{1}^{\prime}=\frac{\frac{2}{T_{1}}+\left(1-\frac{T_{2}}{T_{1}}\right) b_{1}-2 T_{2} b_{0}}{1-b_{1} T_{2}+b_{0} T_{2}^{2}} \\
& b_{0}^{\prime}=\frac{\frac{1}{T_{1}^{2}}+\frac{b_{1}}{T_{1}}+b_{0}}{1-b_{1} T_{2}+b_{0} T_{2}^{2}} .
\end{aligned}
$$

Case of a First-Order Section:

With $S=s / \omega_{0}$ we write for a first-order section:

$$
H(S)=\frac{a_{1} S+a_{0}}{S+b_{0}} .
$$

Replacing $H_{\text {id }}(S)$ by $H_{\text {ni }}(S)$ from (12) we obtain

$$
H^{\prime}(S)=\frac{a_{1}^{\prime} S+a_{0}^{\prime}}{S+b_{0}^{\prime}} .
$$

Using the same conventions as for the biquad case we find

$$
a_{1}^{\prime}=\frac{a_{1}-a_{0} T_{2}}{1-b_{0} T_{2}} ; \quad a_{0}^{\prime}=\frac{a_{0}+\frac{a_{1}}{T_{1}}}{1-b_{0} T_{2}} ; \quad b_{0}^{\prime}=\frac{b_{0}+\frac{1}{T_{1}}}{1-b_{0} T_{2}} .
$$

For $T_{1} \gg 1$ and $T_{2} \ll 1$ we find small sensitivities of pole and zero frequencies and dc-gain with respect to $T_{1}$ and $T_{2}$.

\section{ACKNOWLEDGMENT}

The authors wish to thank R. F. Wassenaar and B. Nauta for their comments and correction of the text.

\section{REFERENCES}

[1] C. F. Chiou and R. Schaumann, "Design and performance of a fully integrated bipolar $10.7 \mathrm{MHz}$ analog bandpass filter," IEEE J. Solid-State Circuits, vol. SC-21, pp. 6-14, Feb. 1986.
[2] A. P. Nedungadi and R. L. Geiger, "High frequency voltage-controlled continuous-time lowpass filter using linearised CMOS integrators," Eleccontinuous-time lowpass filter using linearise

[3] H. Khorramabadi and P. R. Gray, "High frequency CMOS continuoustime filters," IEEE J. Solid-State Circuits, vol. SC-19, pp. 939-948, Dec. 1984.

[4] J. M. Khoury and Y. P. Tsividis, "Analysis and compensation of highfrequency effects in integrated MOSFET-C continuous-time filters," IEEE Trans. Circuits Syst., vol. CAS-34, pp. 862-875, Aug. 1987.

[5] K. W. Moulding, J. R. Quartly, P. J. Rankin, and R. S. Thompson, "Gyrator video IC with automatic tuning," IEEE J. Solid-State Circuits, vol. SC-15, pp. $963-967$, Dec. 1980

[6] F. Krummenacher and N. Joehl, "A 4-MHz CMOS continuous-time filter with on-chip automatic tuning," IEEE J. Solid-State Circuits, vol. 23. pp. $750-758$, June 1988 .

[7] C. S. Park and R. Schaumann, "Design of a 4-MHz analog integrated CMOS transconductance-C bandpass filter," IEEE J. Solid-State Circuits, vol. 23, pp. 987-996, Aug. 1988

[8] H. Hagiwara, M. Kumazawa, S. Takagi, M. Furihata, M. Nagata, and T. Yanasigawa, "A monolithic video frequency filter using NIC-based gyrators," IEEE J. Solid-State Circuits, vol. 23, pp. 172-182, Feb. 1988

[9] M. S. Ghausi and K. R. Laker, Modern Filter Design. Englewood Cliffs, NJ: Prentice-Hall, 1981.

[10] J. R. Brand and R. Schaumann, "Active R filters: Review of theory and practice," IEE J. Electron. Circuits and Systems, vol. 2, pp. 89-101, July 1978.

11] A. S. Sedra and P. Brackett, Filter Theory and Design: Active and Passive. Beaverton, OR: Matrix, 1978

\section{High-Frequency and High- $Q$ Tunable Active Filters}

\section{YANNICK DEVILLE}

Abstract - An investigation of the performance of classical second-order bandpass active filters shows that since they use low-gain elements, they can be divided into two classes at high frequencies: low-selectivity stable filters and low-stability selective filters. This paper presents structures that realize a good compromise between both classes, so that high $Q$ can be achieved with good stability. Verifications have been carried out with experimental Si hybrid high-frequency filters and with computer simulations of GaAs microwave integrated filters. The center frequency of the GaAs filters is tuned with MESFET's used as voltage-controlled resistors and can exceed $2 \mathrm{GHz}$.

\section{INTRODUCTION}

At low frequencies, continuous-time active filters can combine high selectivity and stability by using very high-gain operational amplifiers (op amps). However, the gain of high-frequency and microwave amplifiers is limited by stability considerations that are developed hereafter. We will show that second-order bandpass filters using finite-gain amplifiers, which are our main concern here, can be divided into two classes, according to their quality factor $Q$ defined as the ratio of the center frequency to the 3-dB bandwidth:

1) Class $A$ : filters that are stable $(Q>0)$ whatever their element values are, but that achieve low selectivity when the use limited values for the voltage gain $K$ :

$$
Q_{\max }=\frac{1}{2} \cdot \sqrt{K+1}
$$

Manuscript received August 23, 1988; revised January 4, 1989. This paper was recommended by Associate Editor T. T. Vu.

The author is with the Laboratoires d'Electronique et de Physique Appliquée. 94451 Limeil-Brevannes Cedex, France.

IEEE Log Number 8928279 